Apidologie, 1976, 7 (2), 189-195.

\title{
FLUCTUATIONS DE POPULATIONS NATURELLES CHEZ LES GENRES CHELOSTOMA ET HERIADES (HYMENOPTERA MEGACHILIDAE)
}

\section{Natürliche Populationsschwankungen bei den Arten Chelostoma und Heriades (Hymenoptera Megachilidae)}

\author{
Maria de Lourdes MACIEL DE ALMEIDA CORREIA * \\ Laboratoire d'Éthologie et d'Écologie des Insectes, I.N.R.A., \\ 86600 Lusignan
}

\author{
SUMMARY \\ VARIATIONS OF NATURAL POPULATIONS OF THE GENUS \\ CHELOSTOMA AND HERIADES (HYMENOPTERA MEgaCHILIDAE)
}

Populations of Chelostoma and Heriades in the area of Lusignan ** were analysed.

Both insects do not have the same monthly distribution; however it seems that the distribution of each of them is dependent upon the respective level of their present populations.

The observations on the flight periods of both insects lead to the following remark : in 1970-1972 a greatest number of Heriades nests was observed only when Chelostoma had a low nesting activity. These observations seem to be confirmed in the following years (1973-1974), during which the very low population level of Chelostoma seems to have no effect upon the nesting activity of Heriades.

\section{RÉSUMÉ}

Des populations de Chelostoma et d'Heriades ont été analysées dans la région de Lusignan.

Les deux insectes n'ont pas la même répartition mensuelle, cependant dans les deux cas il semble que cette répartition soit conditionnée pour l'un et pour l'autre genre par le niveau respectif de leurs populations simultanément présentes.

* Boursière de la Fondation Calouste Gulbenkian.

** Town located in the Middle West of France. 
Les observations sur les périodes de vols de ces deux insectes nous conduisent à remarquer que pour les années 1970-1972 c'est seulement lorsque Chelostoma a une activité de nidification réduite qu'un nombre maximum de nids d'Heriades est observé. Ces observations semblent se confirmer les années suivantes (1973-1974) pendant lesquelles le niveau très bas de population de Chelostoma ne semble avoir aucune influence sur l'activité de nidification d'Heriades.

\section{INTRODUCTION}

Nous présentons ici les premiers résultats concernant l'analyse des populations de Chelostoma et d'Heriades (le premier genre représenté par C. nigricornis $\mathbf{N y l}$., le deuxième par $H$. truncorum $\mathrm{L}$. et $H$. crenulatus $\mathrm{Nyl}$.); ces abeilles solitaires palearctiques appartiennent à la famille des Megachilidae et nidifient dans les mêmes sites naturels. Il est bon de souligner qu' $H$. crenulatus à tendance méridionale ne représente que le $1 / 4$ des effectifs d'Heriades. Les deux genres sont voisins et ont été longtemps considérés par les systématiciens européens comme étant un seul genre $=$ Hériades. Au cours de notre travail nous utiliserons les seuls noms de genre puisque au sein de la population d'Heriades existent deux espèces qu'il est difficile de distinguer.

Le premier genre construit ses bouchons et ses cloisons en terre et récolte du pollen de Campanulacées, le deuxième édifie son nid avec une substance résineuse et récolte du pollen de Composées.

Dans la région de Lusignan ${ }^{1}$ ces deux genres se rencontrent en assez grande abondance. C'est pourquoi l'étude de ces insectes a pu être entreprise.

Ces deux genres ont surtout intéressé les systématiciens et à notre connaissance il existe peu de travaux concernant leur biologie.

Nous avons observé les fluctuations de populations de ces deux genres au cours de 5 années de 1970 à 1974 dans leur milieu naturel : période de nidification, variabilité mensuelle et annuelle.

\section{MATÉRIEL ET MÉTHODES}

Les sites d'observation ayant des facteurs climatiques semblables ont été choisis dans un rayon de $40 \mathrm{~km}$ autour de Lusignan.

La méthode de piègeage à l'aide de nichoirs à bambous est celle qui est employée par TASEI (1972). Les nichoirs sont accrochés à de vieux murs, vieux troncs d'arbres, poteaux en bois et charpentes exposées au soleil.

Des visites hebdomadaires sont effectuées à chaque lieu d'échantillonnage de façon à contrôler les débuts de construction et les étapes du développement. Les tubes complètement remplis (nids terminés) sont remplacés par des tubes de bambous vides, à chaque visite et une vérification des bambous restant est également effectuée.

Dans le but d'étudier le comportement des deux insectes Chelostoma et Heriades qui utilisent les nichoirs, nous avons entrepris une analyse approfondie. L'étude porte sur cinq ans et et les comptages de nids ont été effectués entre juin et octobre, mois d'activité de ces abeilles ${ }^{2}$.

1. Ville située dans le Centre-Ouest de la France à $28 \mathrm{~km}$ de Poitiers.

2. Les données concernant les années 1970 à 1973 ont été fournies par l'équipe de travail de TASEI. 


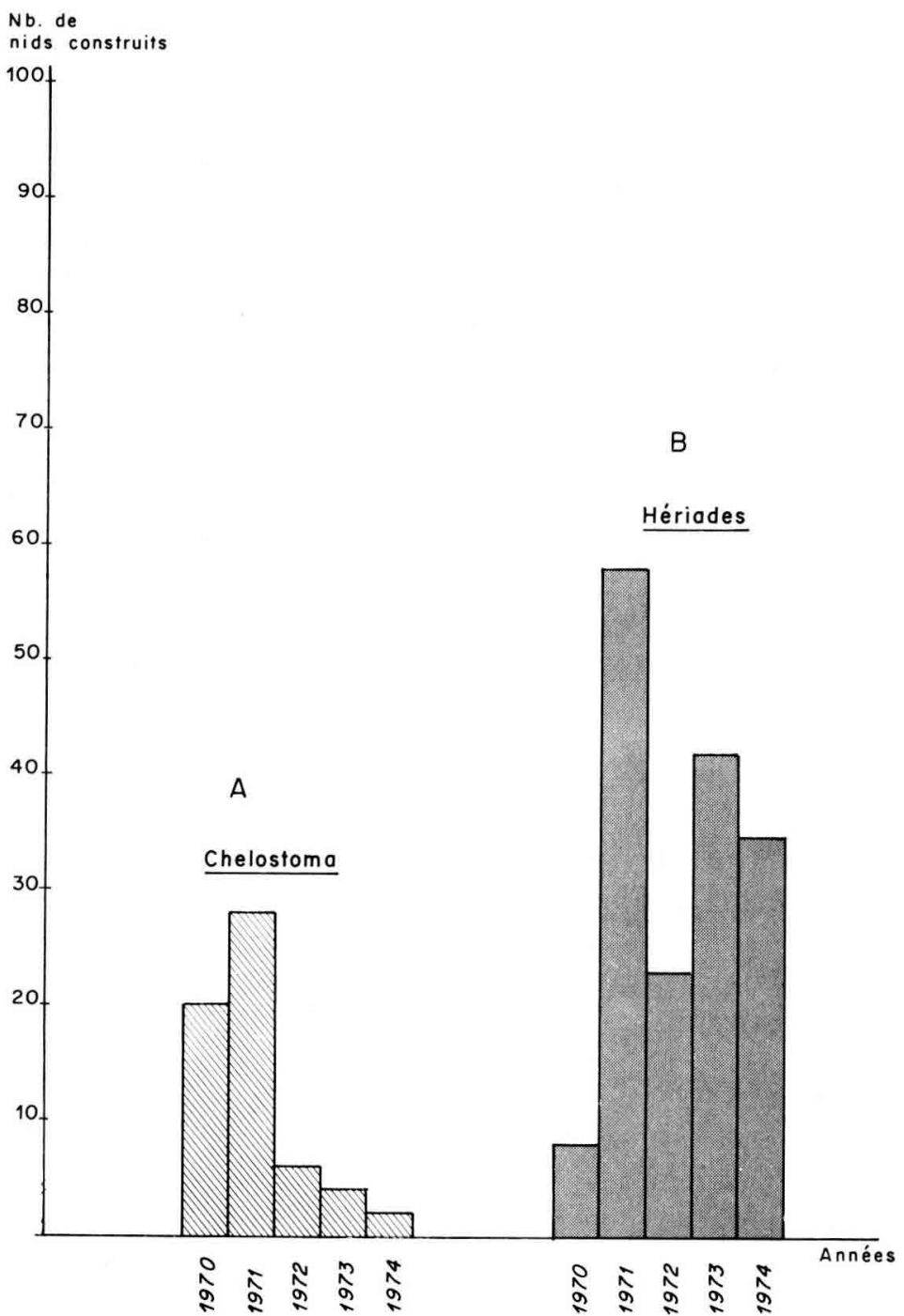

Fig. 1. - Abondance des genres Chelostoma et Heriades à Lusignan durant la période 1970 à 1974. A - population de Chelostoma.
B - population d'Heriades.

Verticalement : nombre de nids construits

Aвв. 1. - Das Vorkommen der Arten Chelostoma und Heriades in Lusignan in den Jahren 1970-1974.

A -.- Population von Chelostoma.

$\mathrm{B}=$ Population von Heriades.

Ordinate : Zahl der gebauten Nester 


\section{RÉSULTATS}

Données générales sur l'évolution des populations de Chelostoma et Heriades au cours de cinq années

\section{Variations annuelles des populations de Chelostoma et d' Heriades}

Après une première analyse des nidifications obtenues (60 nids de Chelostoma et 166 d'Heriades) on constate que les deux populations évoluent de façon différente au cours de la période étudiée. En effet, tandis que les populations de Chelostoma semblent passer par un maximum en 1971 puis régresser progressivement (fig. $1 \mathrm{~A}$ ), celles d'Heriades évoluent de ma.ıière moins régulière et paraissent en augmentation (fig. $1 \mathrm{~B}$ ). Signalons que les nichoirs ne sont jamais entièrement occupés par les abeilles. En général, ce sont seulement 2 à $3 \%$ des tubes offerts qui sont occupés. Dans quelques cas un maximum de $23 \%$ est atteint.

Variations mensuelles de Chelostoma et d'Heriades : observations relatives aux périodes de vol

L'interprétation de la figure 2 nous permet de conclure que les deux espèces sont monovoltines. Au sein de leur habitat, elles apparaissent pendant la même période de l'année (tabl. 1); toutefois la période de vol d'Heriades (Juin à Octobre) est plus longue que celle de Chelostoma (Juin à Septembre). La présence de Chelostoma est très importante en Juillet et Août, celle d'Heriades en Août et Septembre. La population du premier genre nidifie en moyenne plus précocement que celle du deuxième.

\section{DISCUSSION ET CONCLUSION}

Chaque année d'expérimentation, le nombre de nids construits par Heriades et Chelostoma augmente entre juin et août, passe par un maximum en juillet-août et diminue ensuite. Le nombre de nids construits par Heriades est toujours plus grand que celui de Chelostoma. La présence du premier genre est très importante entre août et septembre, celle du deuxième entre juillet et août. Les conditions de nidification optimales varient probablement d'un genre à l'autre et le degré d'influence de chacun des facteurs climatiques fera l'objet d'une étude ultérieure (M. Correia, note non publiée).

Le niveau de chacune des deux populations varie d'une année à l'autre. Lorsqu'un des genres est observé en abondance, l'autre devient de plus en plus rare. 


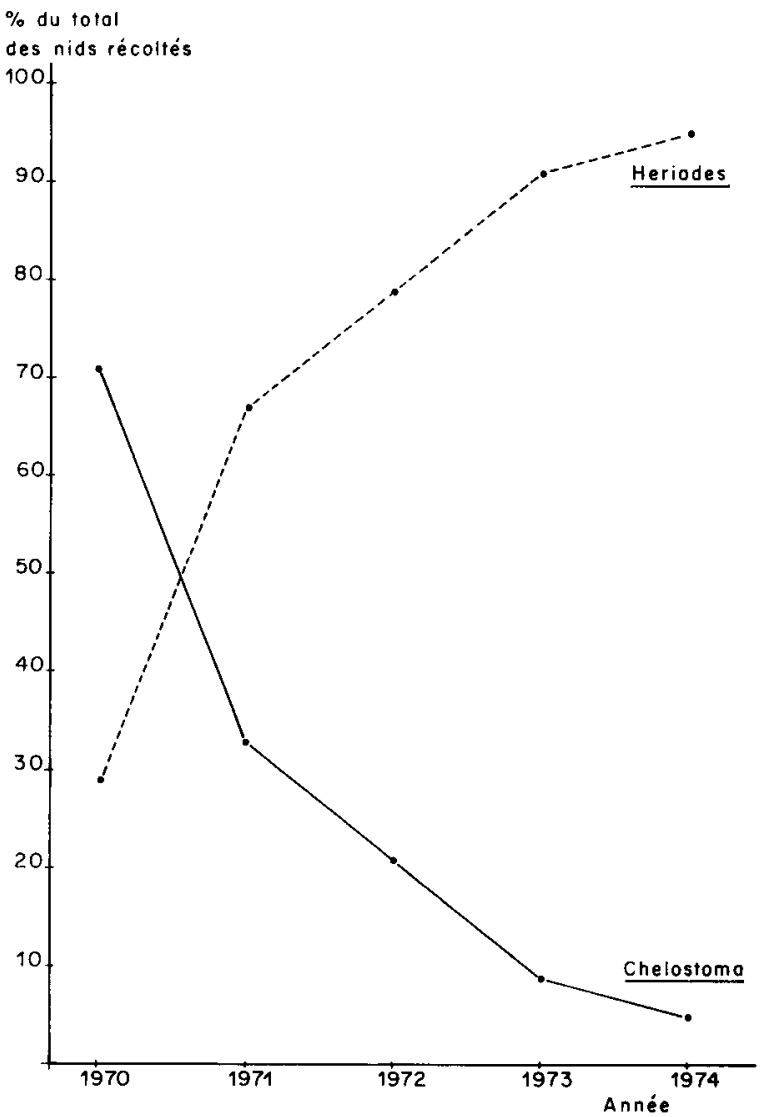

FIG. 2. - Abondance relative d'Heriades et de Chelostoma à Lusignan. Verticalement : Pourcentage du total des nids récoltés.

$$
\text { - Chelostoma }
$$

Aв8. 2. - Relative Häufigkeit von Heriades und Chelostoma in Lusignan. Ordinate : Prozentsatz der gesamten eingesammelten Nester.

$$
\text { Chelostoma. }
$$

TABL. 1. - Nombre de nids fondés chaque mois par Chelostoma et Heriades de 1970 à 1974.

\begin{tabular}{|c|c|c|c|c|c|c|}
\hline Espèce & Juin & Juillet & Août & Septembre & Octobre & Total \\
\hline Chelostoma .......... & 1 & 24 & 27 & 8 & 0 & 60 \\
\hline Heriades ............. & 1 & 23 & 95 & 43 & 4 & 166 \\
\hline $\begin{array}{l}\text { Total } \ldots \ldots \cdots \cdots \cdots \cdots \\
\text { Gesant }\end{array}$ & 2 & 47 & 122 & 51 & 4 & 226 \\
\hline
\end{tabular}

TAB. 1. - Zahl der in den Jahren 1970-1974 je Monat von Chelostoma und Heriades gebauten Nester. 

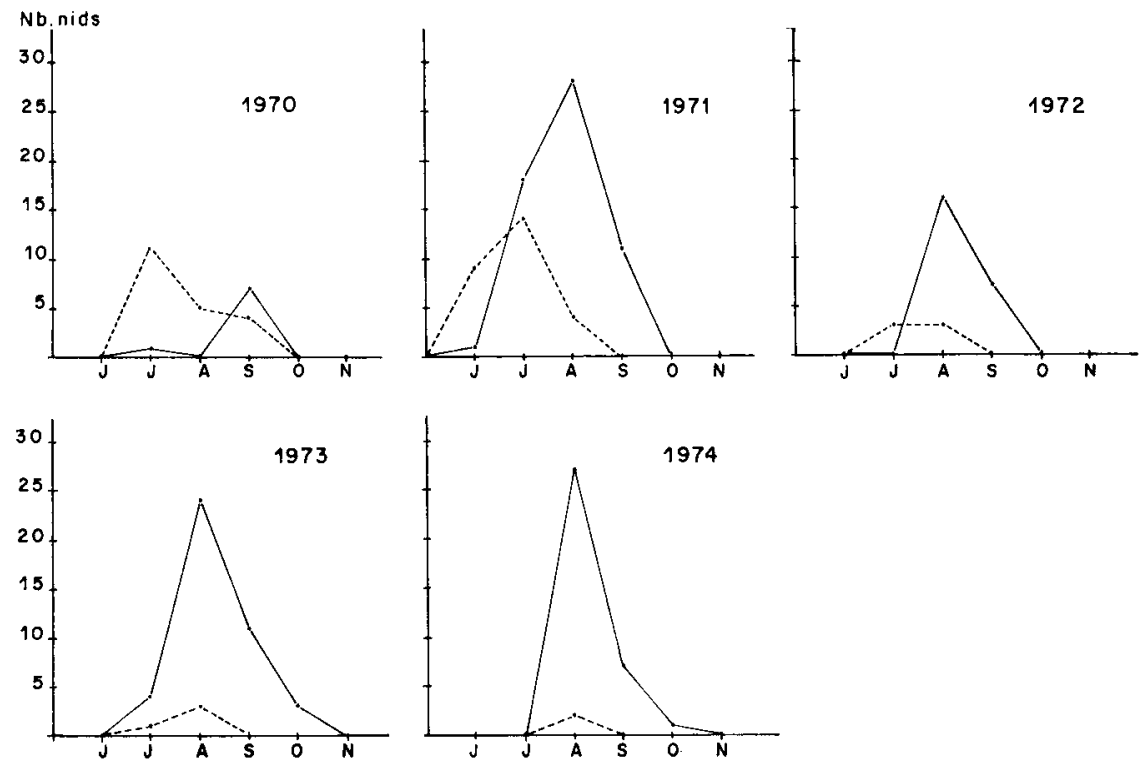

FIG. 3. - Variations mensuelles du nombre de nidifications de 1970 à 1974 chez Chelostoma et Heriades à Lusignan.

\section{- Heriades. \\ _ _ Chelostoma.}

Verticalement : Nombre de nids construits.

Авв. 3. - Monatliche Veränderungen in der Zahl der Nistanlagen von Chelostoma und Heriades in den Jahren 1970-1974 in Lusignan.

- Heriades.

Ordinate : Zahl der gebauten Nester.

S'agit-il du résultat d'une action de facteurs écologiques sur les niveaux respectifs des deux insectes? Seules des observations complémentaires sur ces deux insectes dans un même habitat contrôlable expérimentalement peuvent apporter une réponse à cette question.

Reçu pour publication en janvier 1976.

Eingegangen im Januar 1976.

\section{ZUSAMMENFASSUNG}

In den Jahren 1970-1974 wurden in der Gegend von Lusignan ${ }^{1}$ mit Hilfe von künstlichen Nistgelegenheiten eine Anzahl von Heriades und Chelostoma eingefangen.

1. Stadt im Mittelwesten Frankreichs, $28 \mathrm{~km}$ von Poitiers entfernt. 
Eine erste Analyse der erhaltenen Nestbauten ergab, dass die Stärke beider Populationen von Jahr zu Jahr verschieden ist. Auch die Verteilung über die Monate eines Jahres ist bei beiden Insekten nicht die gleiche. Chelostoma ist durchschnittlich früher als Heriades.

Es scheint indessen, dass in beiden Fällen die jeweilige, gleichzeitig vorhandene Volksstärke die Verteilung beider Arten bedingt. Unsere Untersuchungsergebnisse zeigen, dass bei Chelostoma die Verteilung in den verschiedenen Jahren die gleiche ist, mit anderen Worten : weder das Datum noch der frühzeitige Beginn des Nestbaues wird von den Gegebenheiten eines Jahres beeinflusst. Heriades weist eine von Jahr zu Jahr schwankende Verteilung auf. Besonders 1971 begannen die Nestbauten sehr früh, zur gleichen Zeit wie bei Chelostoma.

Es wurde auch festgestellt, dass während der vier Versuchsjahre die Anzahl der gebauten Nester zwischen Juni und August zunimmt, im August das Maximum erreicht und von da ab stetig geringer wird.

Die Zahl der von Heriades gebauten Nester ist stets grösser als die von Chelostoma. Die Flugzeit von Heriades erstreckt sich über einen längeren Zeitraum als die von Chelostoma. Heriades fliegt zwischen Juni und Oktober, Chelostoma von Juni bis September.

Der Vergleich der Tabellen (Abb. 1 u. 2) deutet auf das Vorhandensein verschiedener Faktoren hin, die die Stärke der Populationen beider Arten beeinflussen. Er zeigt aber auch eine relative Verschiebung oder das Verschwinden von Chelostoma im Verhältnis zu Heriades auf, sowie gemeinsame Faktoren (gleiche Maxima).

Der Einfluss ökologischer oder anderer Faktoren auf die jeweilige Stärke der Populationen beider Arten sollte näher untersucht werden. Nur ergänzende Beobachtungen beider Insekten in der gleichen, experimentell zu kontrollierenden Umgebung kann genauere Erkenntnisse zu unseren Studien liefern.

\section{RÉFÉRENCES BIBLIOGRAPHIQUES}

Benoist R., 1929, Les Hériades de la faune française (Hymenoptera Apidae). Ann. Soc. Ent. Fr., 98, 131-141.

Michener C.D., 1938, American bees of the genus Heriades. Ann. Ent. Soc. Amer., 31, 514-531. TASEI J.-N., 1972, Observations préliminaires sur la biologie d'Osmia coerulescens L. pollinisateur de la luzerne. Apidologie, 3, 149-165. 